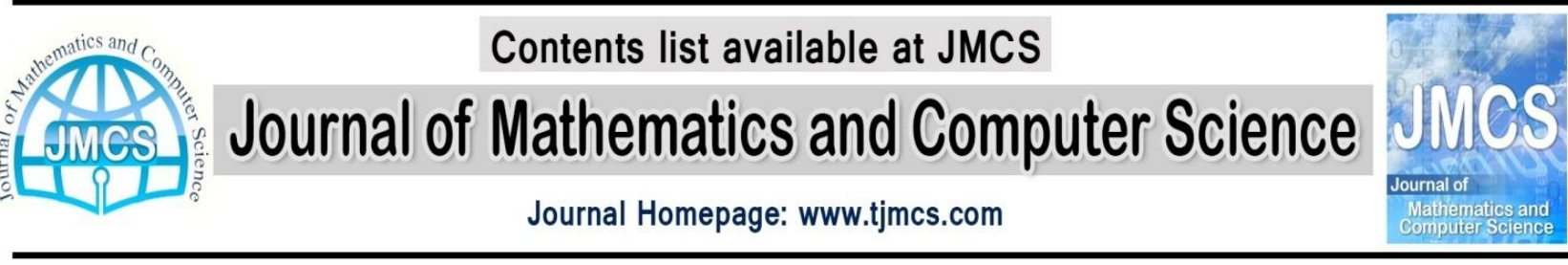

\section{Optimization of Orthogonal Polyphase Coding Waveform for MIMO Radar based on Evolutionary Algorithms}

\author{
Hamed Azami ${ }^{1}$, Milad Malekzadeh ${ }^{2}$, Saeid Sanei ${ }^{3}$ and Alireza Khosravi ${ }^{4}$ \\ ${ }^{1}$ Department of Electrical Engineering, Iran University of Science and Technology \\ hamed_azami@ieee.org \\ ${ }^{2}$ Department of Electrical and Computer Engineering, Babol University of Technology \\ m.malekzade@stu.nit.ac.ir \\ ${ }^{3}$ Faculty of Engineering and Physical Sciences, University of Surrey, UK, \\ s.sanei@surrey.ac.uk \\ ${ }^{4}$ Faculty of Electrical and Computer Engineering, Babol University of Technology \\ akhosravi@nit.ac.ir
}

\begin{abstract}
Using multiple antennas at both transmitter and receiver to improve communication performance is referred to as multi-input multi-output (MIMO) system. In order to keep away interference and increase the independency between the information received from or reflected by various targets, the transmitted signals are required to be mutually orthogonal. In this paper a new approach using evolutionary algorithms including particle swarm optimization (PSO), bee algorithm (BA) and artificial bee colony $(\mathrm{ABC})$ to design orthogonal discrete frequency coding waveforms (DFCWs) is proposed. These methods have desirable autocorrelation and cross correlation characteristics for orthogonal MIMO radars. The simulation results and comparisons demonstrate that each evolutionary algorithm has its own advantages and disadvantages and therefore can be applied to meet particular requirements.
\end{abstract}

Keywords: Poly phase, MIMO radars, and evolutionary algorithms. 


\section{Introduction}

Multi-input multi-output radar (MIMO) is extremely useful in communication science in two decades ago. The MIMO radar is a multiple antenna radar system which is capable to transmit arbitrary waveforms from each antenna element. The multiple transmitting antennas transmit orthogonal signals while multiple receiving antennas receive them $[1,2]$.

The MIMO radar technology has rapidly paid attention from many researchers. Several advantages of the MIMO radar have been discovered by many different researchers such as increased diversity of the target information, excellent interference rejection capability, ability of parameters identification and enhanced flexibility for transmitting beam pattern design. However, the ability to detect the low speed target on the background of clutter and the detection ability of weak target on the background of strong clutter cause that MIMO radar performance has been considered in different projects [3].

According to the MIMO radars' structure, there are different transmitted signal, so receiving the transmitted signal without interference is the most important purpose in this application. In order to overcome this problem, the transmitted signals must be mutually orthogonal [4].

The MIMO radar uses $L$ orthogonal waveforms that are transmitted from different phase centers and $N$ shows the receiving phase centers. The received signals are matched filtered for each of the transmitted waveforms forming $N L$ channels. This assumption denotes the necessity of low cross correlation properties between waveforms. In addition to have high resolution for multiple target detection, a low auto-correlation sidelobe peaks levels for transmitted signals is required [5].

Assume a MIMO radar system that composes of $L$ transmitters, where each of them transmits a distinct signal from an orthogonal code set $\left\{s_{l}(t), l=1,2,3, \ldots, L\right\}$ in which any two signals in the set are uncorrelated and the aperiodic autocorrelation function of any code $s_{l}(t)$ should be closed to an impulse function.

The capability of being achieved of MIMO radars relates to the feasibility of a set of orthogonal signals with low autocorrelation and cross correlation properties. As a consequence, the acceptable design of such orthogonal code sets is very important for putting into effective MIMO radar systems [6]. MIMO radar systems can be coded with binary sequences, polyphase sequences, or frequency-hopped sequences. The polyphase code has some advantages against the binary code; as might be expected, polyphase code is increasingly becoming a desirable alternative for radar signals [7].

In this paper, we intend to demonstrate powerful nature inspired algorithms, PSO, new PSO (NPSO), BA and $\mathrm{ABC}$ for the designing of orthogonal ployphase code sets that can be used in MIMO radars. The remainder of this paper is organized as follows: in the second section, the problem of the polyphase code set design is presented. Then, four kinds of nature inspired algorithms to numerically optimize polyphase code sets are introduced briefly. The results from designing methods are presented in section 4. Finally, the last section concludes the paper. 


\section{Orthogonal Polyphase Signal Design for MIMO}

Consider that orthogonal polyphase code comprise $L$ signals that each signal containing $N$ subpulses represented by a complex number sequence, the signal set can be shown as follows:

$s_{l}(t)=e^{j \varphi_{l}(n)}, n=1,2, \ldots, N, l=1,2, \ldots, L$

where $\varphi_{l}(n),\left(0 \leq \varphi_{l}(n) \leq 2 \pi\right)$ is the phase of subpulse $n$ of signal $L$ in the signal set [1].

Assume a polyphase code set $s$ with code length of $N$, set size of $L$, one can concisely represent the phase values of $s$ with the following $L \times N$ phase matrix:

$$
s(L, N)=\left[\begin{array}{cccc}
\varphi_{1}(1) & \varphi_{1}(2) & \ldots & \varphi_{1}(n) \\
\varphi_{2}(1) & \varphi_{2}(2) & \ldots & \varphi_{2}(n) \\
\vdots & \vdots & \ddots & \vdots \\
\varphi_{L}(1) & \varphi_{L}(2) & \ldots & \varphi_{L}(n)
\end{array}\right]
$$

where the phase sequence in row $l(1 \leq l \leq L)$ is the polyphase sequence of signal $l$, and all the elements in the matrix can only be chosen from the phase set in (2).

From the autocorrelation and cross correlation characteristic of orthogonal polyphase codes, we get:

$A\left(\varphi_{l}, k\right)=\left\{\begin{aligned} & \frac{1}{N} \sum_{n=1}^{N-k} \exp j\left[\varphi_{l}(n)-\varphi_{l}(n+k)\right]=0, 0<k<N \\ & l=1,2, \ldots, L \\ & \frac{1}{N} \sum_{n=-k+1}^{N} \exp j\left[\varphi_{l}(n)-\varphi_{l}(n+k)\right]=0,-N<k<0\end{aligned}\right.$

$C\left(\varphi_{p}, \varphi_{q}, k\right)= \begin{cases}\frac{1}{N} \sum_{n=1}^{N-k} \exp j\left[\varphi_{q}(n)-\varphi_{p}(n+k)\right]=0, & 0<k<N \\ \frac{1}{N} \sum_{n=k+1}^{N} \exp j\left[\varphi_{q}(n)-\varphi_{p}(n+k)\right]=0, & -N<k<0\end{cases}$

where $A\left(\varphi_{l}, k\right)$ and $C\left(\varphi_{p}, \varphi_{q}, k\right)$ are the aperiodic autocorrelation function of polyphase sequence $S_{l}$ and the cross correlation function of sequences $s_{p}$ and $s_{q}$, and $k$ is the discrete time index. Therefore, designing an orthogonal polyphase code set is equivalent to the constructing a polyphase matrix in (2) with $A\left(\varphi_{l}, k\right)$ and $C\left(\varphi_{p}, \varphi_{q}, k\right)$ constraints in (3) and (4).

For the design of orthogonal polyphase code sets used in MIMO radar systems, an optimization criterion is not only to minimize the autocorrelation sidelobe peak (ASP) and the cross correlation peaks (CP), but also minimize the total autocorrelation sidelobe energy and cross correlation energy in (3) and (4). The peak and energy based cost function used for MIMO radar signals is defined as follows [8]: 


$$
E=(1-\mu) \sum_{l=1}^{L} \sum_{K=1}^{N-1} \max \left|A\left(\phi_{l}, k\right)\right|^{2}+\mu \sum_{p=1}^{L-1} \sum_{q=p+1}^{L} \sum_{k=-(N-1)}^{N-1}\left|C\left(\phi_{p}, \phi_{q}, k\right)\right|^{2}
$$

\section{Evolutionary Algorithms for DFCW}

In past decades engineers have concentrated to present heuristic methods to solve optimization problems. In this way they have tried to inspire from nature and from this point of view. Finally, they have succeeded to achieve to different evolutionary algorithms including PSO, NPSO, BA and ABC. In this paper, to design the orthogonal discrete frequency coding waveforms, these algorithms are used.

\subsection{Particle Swarm Optimization and New Particle Swarm Optimization}

The idea of PSO was first raised by J. Kennedy and R. Eberhart in 1995 [9]. PSO is an evolutionary computing algorithm inspired by nature and is based on repetition. The social behavioral of animals like birds and fish when they are together has been the inspiration source for this algorithm [10]. PSO, the same as other evolutionary algorithms, begins with a random matrix as a initial population. Unlike genetic algorithms (GA), normal PSO doesn't have evolutionary operators like mutation and breeding. Each member of the population is called a particle. In fact, in the PSO algorithm a certain number of particles that are formed randomly make the initial values. There are two parameters for each particle, namely, position and velocity of the particle, which are defined by a space vector and a velocity vector, respectively. These particles form a pattern in an n-dimensional space and move to the desired value. The best position of each particle in the past and the best position among all particles are stored separately. According to the experience from the previous moves, the particles decide how to make the next move. In every iteration, all particles in the $n$-dimensional problem space move to an optimum point. In each iteration, the position and velocity of each particle can be modified according to the following equations:

$$
\begin{aligned}
& v_{i}(t+1)=w v_{i}(t)+C_{1} r_{1}\left(p_{\text {best }}(t)-x_{i}(t)\right)+C_{2} r_{2}\left(g_{\text {best }_{i}}(t)-x_{i}(t)\right) \\
& x_{i}(t+1)=x_{i}(t)+v_{i}(t+1)
\end{aligned}
$$

where $n$ represents the dimension $(1 \leq n \leq N), C_{1}$ and $C_{2}$ are positive constants, generally considered 2.0. $r_{1}$ and $r_{2}$ are random numbers uniformly between 0 and $1 ; w$ is inertia weight that can be constant or defined by equation [11].

Equation (6) expresses that the velocity vector of each particle is updated $\left(v_{i}(t+1)\right)$ and the new and previous values of the vector position $\left(x_{i}(t)\right)$ create the new position vector $\left(x_{i}(t+1)\right)$. In fact, the updated velocity vector affects both local and global values. The best response of the local positions is the best solution of the particle until current execution time $\left(p_{\text {best }}\right)$ and the best global solution is the best solution of the entire particles until current execution time $\left(g_{\text {best }}\right)$.

Since PSO stay in local minimums of fitness function we use NPSO. In each iteration, as was said in PSO, global best particle and local best particle are computed. NPSO strategy uses the global best particle and local "worst" particle, the particle with the worst fitness value of the particle until current execution time [12]. It can be defined as: 
$v_{i}(t+1)=w v_{i}(t)+C_{1} r_{1}\left(p_{\text {worst }}(t)-x_{i}(t)\right)+C_{2} r_{2}\left(g_{\text {best }}(t)-x_{i}(t)\right)$

\subsection{Artificial Bee Colony Algorithm}

$\mathrm{ABC}$ is one of the most recently defined algorithms proposed by Karaboga and Bastutk in 2006, is inspired by the intelligent behavior of honeybees. In the $\mathrm{ABC}$ algorithm, the colony of artificial bees contains three groups of bees: employed, onlookers and scouts. This algorithm has been shown at 9 steps as follows:

- Initialize the population of solutions

- Evaluate the population

- Produce new solutions for the employed bees

- Apply the greedy selection process

- Calculate the probability values

- Produce the new solutions for the onlookers

- Apply the greedy selection process

- Determine the abandoned solution for the scouts, and replace them with a new randomly produced solutions

- Memorize the best solution achieved so far

In this algorithm, first half of the colonies are selected as the employed artificial bees and the rest of them are chosen as the onlookers. For each food source, there is only one employed bee. In other words, the number of employed bees is equal to the number of food sources around the hive. Scout is an employed bee whose food source has been abandoned. Generally, the ABC algorithm consists of local and global searches to find an optimum answer in desired space. In this algorithm, the position of a food source represents a possible solution to the optimization problem and the nectar amount of a food source corresponds to the quality or fitness of the associated solution. The number of the employed or the onlooker bees is equal to the number of solutions in the population. An onlooker bee assesses the information of the nectar taken from all employed bees and selects a food source with a probability $p_{i}$ related to its fitness value [13].

$p_{i}=\frac{f i t_{i}}{\sum_{n=1}^{S N} f i t_{n}}$

where $S N$ and $f i t_{i}$ are the size of population and the fitness value of the solution $i$ which is proportional to the nectar amount of the food source in the position $i$, respectively. In order to produce a candidate food position from the old one in memory, the $\mathrm{ABC}$ uses the following expression:

$v_{i j}=x_{i j}+\phi_{i j}\left(x_{i j}-x_{k j}\right)$

where $\phi_{i j}$ denotes a random number and selected between $[-1,1]$ and $k \in\{1,2, \ldots, S N\}$ and $j \in\{1,2, \ldots, D\}$ are randomly selected indexes. Each solution $x_{i}(i=1,2, \ldots, N)$ is represented by a $D$-dimensional vector, where $D$ denotes the number of parameters to be optimized and each parameter is real coded [13]. 


\subsection{Bees Algorithm Optimization}

The BA which imitates the food foraging behavior of honey bee colony is a novel swarm-based search algorithm developed by D.T. Pham [14]. This algorithm is based on a kind of neighborhood search combined with random search and can be used for multi-objective optimization. The performance of this algorithm has been shown at 8 steps as follows:

- Initialize population with random solutions

- Evaluate fitness of the population

- While (stopping criterion not met). Forming new population.

- Select sites for neighborhood search.

- Recruit bees for selected sites (more bees for best e sites) and evaluate finesses.

- Select the fittest bee from each patch.

- Assign remaining bees to search randomly and evaluate their fitnesses.

- End While

The optimization of DFCW with BA is summarized as follows:

At the first step the initial phase are produced by random solution. Then, according to the equation (5) we evaluate the fitness function. In order to minimize the fitness function; It is sorted in descending form. Now the $m$ sites and best sites ( $e$ out of $m$ ) are selected with respect to fitness. The recruited bees investigate the selected sites. New values are produced with this equation:

$u(i)=(x(i)-n g h)+(2 * n g h \cdot \operatorname{rand}(\operatorname{size}(x(i), 1), 1))$

According to new value, the new fitness function would be derived. After comparing this fitness with old fitness and repeating this cycle, the BA processing will be done [15].

\section{Simulation Results}

In this section, according to the described optimization algorithm we can design many different lengths of sequences. However, in this paper we present only the correlation properties of sequences of the three code sequences with length $N=128$ and $L=3$. Tables 1,2,3 and 4 list the three code sequences with mentioned parameters for optimizing by PSO, NPSO, BA and ABC, respectively. By comparing Table 1 and 2, it is realized that the performance of NPSO is better than PSO regarding the cross correlation and auto correlation. Also, comparison of Tables 2 and 4 shows that the performance of the NPSO is too much better than ABC. As can be seen in Tables 3 and 4, to design the CPs, applying of BA is better than $\mathrm{ABC}$, while for designing the ASPs applying of $\mathrm{ABC}$ is better than $\mathrm{BA}$. It should be mentioned that the best algorithms to design a CP and an ASP are BA and NPSO, respectively.

Table 1. ASPs and CPs of the designed DFCW set with $N=128$ and $L=3$ using PSO

\begin{tabular}{|c|c|c|c|}
\hline \multicolumn{1}{|c}{ CODE 1 } & CODE 2 & CODE3 \\
\hline CODE1 & 0.1229 & 0.069 & 0.075 \\
\hline CODE2 & 0.069 & 0.1327 & 0.0735 \\
\hline CODE3 & 0.075 & 0.0735 & 0.1186 \\
\hline
\end{tabular}


Table 2. ASPs and CPs of the designed DFCW set with $N=128$ and $L=3$ using NPSO

\begin{tabular}{|c|c|c|c|}
\hline \multicolumn{2}{|c}{ CODE 1 } & CODE 2 & CODE3 \\
\hline CODE1 & 0.1189 & 0.0641 & 0.0601 \\
\hline CODE2 & 0.0641 & 0.1237 & 0.0573 \\
\hline CODE3 & 0.0601 & 0.0573 & 0.1157 \\
\hline
\end{tabular}

Table 3. ASPs and CPs of the designed DFCW set with $N=128$ and $L=3$ using BA

\begin{tabular}{|c|c|c|c|}
\hline & CODE 1 & CODE 2 & CODE3 \\
\hline CODE1 & 0.3835 & 0.0561 & 0.0649 \\
\hline CODE2 & 0.0561 & 0.3224 & 0.0573 \\
\hline CODE3 & 0.0649 & 0.0573 & 0.3032 \\
\hline
\end{tabular}

Table 4. ASPs and CPs of the designed DFCW set with $N=128$ and $L=3$ using ABC

\begin{tabular}{|c|c|c|c|}
\hline & CODE 1 & CODE 2 & CODE3 \\
\hline CODE1 & 0.1254 & 0.1896 & 0.1565 \\
\hline CODE2 & 0.1896 & 0.1227 & 0.1676 \\
\hline CODE3 & 0.1565 & 0.1676 & 0.1657 \\
\hline
\end{tabular}

\section{Conclusions}

MIMO radars can track the target from different angles and it is very important in detection and recognition of the objects in space. In order to enable separation of the information from the targets, the transmitted signal must be orthogonal. This paper has presented an effective approach to design an orthogonal code for the MIMO radars by using four evolutionary algorithms, namely, PSO, NPSO, BA and ABC. This approach demonstrates desirable ASPs and CPs. The simulation results have shown the different abilities of these algorithms. For this application, NPSO has optimized the ASPs and CPs better than PSO and ABC. In order to design acceptable CPs, BA is better than the other algorithms, while for designing the ASPs application of NPSO is better than those of others. It should be mentioned that all of them are attractive optimization algorithms in order to solve this problem with different $L$ and $N$ but they may best perform in different applications.

\section{References}

[1] M. Malekzadeh, A. Khosravi, S. Alighale and H. Azami, "Optimization of Orthogonal Poly phase Coding Waveform based on Evolutionary Algorithms for MIMO Radar", Lecture notes in Computer Science (Springer Verlag), vol. 7389, pp. 95-102 (2012). 
[2] E. Fishler, A. Haimovich, R. Blum, L. Cimini, D. Chizhik, and R.Valenzuela, "MIMO radar: an idea whose time has come", in Proc. of the IEEE Int. Conf. on Radar, Philadelphia (2004).

[3] E. Fishler, A. Haimovich, R. Blum, L. Cimini, D. Chizhik, and R. Valenzuela , "Spatial diversity in radars - models and detection performance", IEEE Trans. on Sig. Proc., vol. 54, pp. 823-838 (2006).

[4] H. Deng, "Polyphase code design for orthogonal netted radar systems", IEEE Transactions on Signal Processing, vol. 52, pp. 3126-3135 (2004).

[5] H. Deng, "Synthesis of binary sequences with good autocorrelationand cross-correlation properties by simulated annealing," IEEE Trans. Aerosp. lectron. Syst., vol. 32, pp. 98-107, Jan. (1996).

[6] E. Grandjean, "Linear time algorithms and NP-complete problems," SIAM J. Comput., vol. 23, no. 3, pp. 573-597 (1994).

[7] B. Liu, Z. He, J. Zeng and B. Liu, "Polyphase orthogonal code design for MIMO radar systems", International Conference on Radar, CIE '06, pp. 1-4 (2006).

[8] B. Liu, Z. He and Q. He, "Optimization of orthogonal discrete frequency-coding waveform based on modified genetic algorithm for MIMO Radar", International Conference on Communications, Circuits and Systems, pp. 966-970 (2007).

[9] L. Mussi, S. Cagnoni, and F. Daolio, "GPU-based road sign detection using particle swarm optimization", International Conference on Intelligent Systems Design and applications, pp. 152-157 (2009).

[10] J. Kennedy and R. Eberhart, "Particle swarm optimization", IEEE Conference on Neural Networks, pp. 1942-1948 (1995).

[11] Z. Rafiee, S. Ganjefar and A. Fattahi, "A new PSS tuning technique using ICA and PSO methods with the fourier transform", Iranian Conference on Electrical Engineering (ICEE), pp. 806-811 (2010).

[12] C. Yang and D. Simon, "A new particle swarm optimization technique", International Conference on Systems Engineering, pp. 164-169 (2005).

[13] D. Karaboga and B. Bastürk, "A powerful and efficient algorithm for numerical function optimization: artificial bee colony (ABC) algorithm”, J. Global Optim., vol. 39, no. 3, pp. 459-471 (2007).

[14] D. T. Pham, A. Ghanbarzadeh, E. Koc, S. Otri, S. Rahim and M. Zaidi, "The bees algorithm, a novel tool for complex optimization problems", International Virtual Conference on Intelligent Production Machines and Systems, pp. 454-459 (2006).

[15] D. T. Pham, A. J. Soroka, E. Koç, A. Ghanbarzadeh, S. Otri and M. Packianather, "Optimising neural networks for identification of wood defects using the bees algorithm", 4th International IEEE Conference on Industrial Informatics, pp. 1346-1351 (2006). 